\title{
Quantum integrability and Bethe ansatz solution for interacting matter-radiation systems
}

\author{
Anjan Kundu \\ Saha Institute of Nuclear Physics, Theory Group \\ 1/AF Bidhan Nagar, Calcutta 700 064, India. \\ email: anjan@tnp.saha.ernet.in
}

August 25, 2018

\begin{abstract}
A unified integrable system, generating a new series of interacting matter-radiation models with interatomic coupling and different atomic frequencies, is constructed and exactly solved through algebraic Bethe ansatz. Novel features in Rabi oscillation and vacuum Rabi splitting are shown on the example of an integrable two-atom Buck-Sukumar model with resolution of some important controversies in the Bethe ansatz solution including its possible degeneracy for such models.

PACS numbers: $02.30 \mathrm{Ik}, 42.50 \mathrm{Pq}, 03.65 \mathrm{Fd}, 32.80$-t
\end{abstract}

The basic physics underlying a variety of important phenomena in interacting matter-radiation (MR) systems, like those in quantum optics induced by resonance interaction between atom and a quantized laser field, in cavity QED rempe8790,raizen89, in trapped ion interacting with its center of mass motion irradiated by a laser beam trap,vogel95 etc., seems to be nicely captured by simple models like Jaynes-Cummings (JC) jc, Buck Sukumar (BS) bss and some of their extensions jç1. Many theoretical predictions based on these models, like vacuum Rabi splitting (VRS) raizen89,VRS, Rabi oscillation and its quantum collapse and revival rempes8790 etc. have been verified in maser and laser experiments. However, for describing physical situations more accurately one has to look for further generalizations of the basic models, like q-deformed BS and JC model qbs, qje, trapped ion (TI) with nonlinear coupling vogel95,ntrap, multi-atom models raizen89,nţrap,natom etc. Nevertheless, while the exact solutions for the JC and the BS models together with their simple multi-atom extensions are known jcexact,jcbethe,bsbethe, the same is no longer true for most of the above generalizations. Moreover, while in known multi-atomic MR models the atoms interact only via the oscillator mode jcbethe,bsbethe with coinciding atomic frequencies (AF), integrable models with explicit inter-atomic couplings have not been proposed. Likewise, though q-deformation, which physically signifies introduction of anisotropy together with specific nonlinearity into the system, was considered for a few MR models qbs, qjc, their multi-atom and integrable variants are not known. Therefore, it is indeed a challenge to find a scheme for generating integrable MR models with the desired properties.

To meet this challenge we construct a general integrable system based on the ancestor Lax operator of kunpprl and generate in a unified way a series of integrable multi-atom MR models with explicit inter-atomic interactions and nondegenerate AF. This includes such new generalizations for JC, BS, TI, etc. models and discovers important integrable q-deformations like qBS, qJC, qTI etc. It is worth 
noting that, our integrable TI model exhibits full exponential nonlinearity without any approximation and multi-atom qBS and qJC models involves quantum group spin operators. Moreover, since our construction is based on a general Yang-Baxter (YB) algebra, together with the generation of various models at its different realizations, we can solve them exactly in a unified way through algebraic Bethe ansatz (BA). Our strategy of construction is to start with a Lax operator by taking it as a combination $T(\lambda)=L^{s}(\lambda) \prod_{j}^{N_{a}} L_{j}^{S}(\lambda)$, with $L^{s}(\lambda)$ linked with the ancestor model of kunprl and $N_{a^{-}}$ number of $L_{j}^{S}(\lambda)$ related to the spin model jcbęthe. By construction it must satisfy the YB equation $R(\lambda-\mu) T(\lambda) \otimes T(\mu)=(I \otimes T(\mu))(T(\lambda) \otimes I) R(\lambda-\mu)$, with mutually commuting set of conserved operators obtained from the expansion $\tau(\lambda)=\operatorname{tr} T(\lambda)=\sum_{a} C_{a} \lambda^{a}$ abga. For standard MR models, as we will see below, the Lax operators are rational type linked with the simplest quantum $R$-matrix of $x x x$ spin chain abुa, while for $q$-deformed models they are trigonometric type related to the $R$-matrix of $x x z$ chain x̧xz. We concentrate first on standard MR models and recall that in the rational case the $2 \times 2$ ancestor Lax operator may be given as

$$
L^{s}(\lambda)=\left(\begin{array}{cc}
c_{1}^{0}\left(\lambda+s^{3}\right)+c_{1}^{1}, & s^{-} \\
s^{+}, & c_{2}^{0}\left(\lambda-s^{3}\right)-c_{2}^{1}
\end{array}\right),
$$

with operators s satisfying a quadratic algebra

$$
\left[s^{+}, s^{-}\right]=2 m^{+} s^{3}+m^{-}, \quad\left[s^{3}, s^{ \pm}\right]= \pm s^{ \pm}, \quad\left[m^{ \pm}, \cdot\right]=0 .
$$

The central elements $m^{ \pm}$are expressed through arbitrary parameters appearing in (11) as $m^{+}=$ $c_{1}^{0} c_{2}^{0}, \quad m^{-}=c_{1}^{1} c_{2}^{0}+c_{1}^{0} c_{2}^{1}$ and as it is easy to see, their different choice reduces (2) to different algebras:

$$
\text { i) } s u(u) \text {, at } m^{+}=1, m^{-}=0, \text { ii) } s u(1,1) \text {, at } m^{+}=-1, m^{-}=0 \text {, }
$$

iii) bosonic, at $m^{+}=0, m^{-}=-1$, iv) canonical, at $m^{+}=m^{-}=0$

and the corresponding limits yield from (11) the respective Lax operators. In case i), (1) reduces simply to the spin Lax operator

$$
L_{j}^{S}(\lambda)=\left(\begin{array}{cc}
\lambda+S_{j}^{z}+c_{j}, & S_{j}^{-} \\
S_{j}^{+}, & \lambda-S_{j}^{z}+c_{j}
\end{array}\right),
$$

Our Lax operator constructed as above would generate the set of all commuting conserved operators, with higher ones containing increasingly higher many-body interactions. The simplest among them is $C_{N_{a}}=s^{3}+\sum_{j}^{N_{a}} S_{j}^{z}$, while the next in the set $\alpha C_{N_{a}-1}$, may be defined as the Hamiltonian of our unified MR system:

$$
\begin{aligned}
H_{M R} & =H_{d}+H_{S s}+H_{S S}, \\
H_{d} & =\omega_{f} s^{3}+\sum_{j}^{N_{a}} \omega_{a j} S_{j}^{z}, \\
H_{S s} & =\alpha \sum_{j}^{N_{a}}\left(s^{+} S_{j}^{-}+s^{-} S_{j}^{+}+\left(c_{1}^{0}+c_{2}^{0}\right) s^{3} S_{j}^{z}\right), \\
H_{S S} & =\alpha \sum_{i<j}\left(\left(c_{1}^{0}+c_{2}^{0}\right) S_{i}^{z} S_{j}^{z}+c_{1}^{0} S_{i}^{-} S_{j}^{+}+c_{2}^{0} S_{i}^{+} S_{j}^{-}\right)
\end{aligned}
$$

Here $H_{S s}$ describes matter-radiation, while $H_{S S}$, matter-matter interactions. $\mathbf{S}_{j}, j=1,2, \ldots, N_{a}$ stand for an array of $N_{a}$ atoms, each with $2 s+1$ levels and satisfy the $s u(2)$ algebra. $\mathbf{s}$ on the other hand 
signifies a radiation or a vibration mode and satisfies more general algebra (2). In (15) the radiation frequency $\omega_{f}$ and the atomic frequencies $\omega_{a j}, j=1,2, \ldots, N_{a}$ are defined through inhomogeneous parameters of the Lax operator as

$$
\omega_{f}=\sum_{j} w_{j}, \quad w_{j}=\alpha\left(c_{1}^{0}-c_{2}^{0}\right) c_{j}, \quad \omega_{a j}=\omega_{f}-w_{j}+\alpha\left(c_{1}^{1}+c_{2}^{1}\right)
$$

Remarkably, the general model (5) reduces to a new series of integrable multi-atom BS, JC and TI models in a unified way at the limits ii), iii) and iv) of (3). For example, case ii) with the choice

$$
c_{1}^{0}=-c_{2}^{0}=1, c_{1}^{1}=c_{2}^{1} \equiv c,
$$

yields from (5) the model

$$
H_{B S}=\omega_{f} s^{3}+\sum_{j}^{N_{a}}\left(\omega_{a j} S_{j}^{z}+\alpha\left(s^{+} S_{j}^{-}+s^{-} S_{j}^{+}\right)\right)+\alpha \sum_{i<j}^{N_{a}}\left(S_{i}^{-} S_{j}^{+}-S_{i}^{+} S_{j}^{-}\right),
$$

which with a bosonic realization of $s u(1,1): \quad s^{+}=\sqrt{N} b^{\dagger}, s^{-}=b \sqrt{N}, s^{3}=N+\frac{1}{2}$ and the spin$s$ operator $\vec{S}=\frac{1}{2} \sum_{k}^{2 s} \vec{\sigma}_{k}$, would represent a new integrable multi-atom BS model with inter-atomic interactions and different atomic frequencies. Note that at $N_{a}=1$, when matter-matter interactions vanish and all AF coincide, (8) recovers the known model bsbęthe. However we solve below exactly through BA the more general case with nonvanishing interatomic couplings and all different AF: $\omega_{a j}$, as defined in (6).

Similarly, a new integrable multi-atom JC model with matter-matter coupling is obtained from the same (5) under reduction iii), consistent with $c_{1}^{0}=\alpha, c_{2}^{0}=0, c_{1}^{1} \equiv c, c_{2}^{1}=-\alpha^{-1}$ and bosonic realization $s^{-}=b, s^{+}=b^{\dagger}, s^{3}=b^{\dagger} b$. We do not present here explicit form of this easily derivable Hamiltonian, which yields the known model jcbęthe only at $N_{a}=1$, when interatomic couplings vanish and all AF become degenerate.

We can generate an integrable TI model with interatomic interactions, again from the same MR model (5) at reduction iv), by fixing the parameter values as $c_{1}^{0}=-1, c_{1}^{1} \equiv c, c_{2}^{0}=c_{2}^{1}=0$ and considering consistent realization through canonical variables as $s^{ \pm}=e^{\mp i x}, s^{3}=p+x$. We present here only its $N_{a}=1$ form by a suitable combination with the other conserved quantity $C_{1}$ :

$$
H_{T I}=\left(\omega_{a}-\omega_{f}\right) S^{z}+S^{z 2}+\alpha\left(e^{-i x} S^{+}+e^{i x} S^{-}\right)+H_{x p}
$$

with $H_{x p}=\frac{1}{2}\left(p^{2}+x^{2}\right)+x p, \vec{S}=\frac{1}{2} \sum_{k} \vec{\sigma}_{k}$, which is a new integrable multi-atom TI model with full exponential nonlinearity without approximation.

For constructing integrable q-deformed MR models the strategy would be the same; only one has to start now from the trigonometric type ancestor Lax operator involving q-deformed operators and associated with $x x z R$-matrix, the explicit form of which is given in kunprl. For simplicity we present here only $N_{a}=1$ case with the Hamiltonian

$$
\begin{aligned}
H_{q M R} & =H_{d}+\left(s_{q}^{+} S_{q}^{-}+s_{q}^{-} S_{q}^{+}\right) \sin \alpha, \\
H_{d} & =-i c_{0} \cos (\alpha X)+c \sin (\alpha X), X=\left(s_{q}^{3}-S_{q}^{z}+\omega\right),
\end{aligned}
$$

which represent a new class of $\mathrm{MR}$ models with $\mathbf{S}_{q}$ belonging to the quantum group $U_{q}(\operatorname{su}(2))$ and $\mathbf{s}_{q}$ to a more general quantum algebra kunsprl. It is important to note, that $\mathbf{s}_{q}$ can yield a variety of 
q-deformed operators, inducing (10) to generate a number of physically relevant q-deformed integrable MR models.

For example, an integrable q-deformed $B S$ model may be constructed from (10) at $c_{0}=0$, by realizing $\mathbf{s}_{q}$ through q-oscillator: $s_{q}^{+}=\sqrt{[N]_{q}} b_{q}^{\dagger}, s_{q}^{-}=b_{q} \sqrt{[N]_{q}}, s_{q}^{3}=N+\frac{1}{2}$, and quantum spin operator $\mathbf{S}_{q}$ by using its co-product x̧xz : $S_{q}^{ \pm}=\sum_{j}^{s} q^{-\sum_{k<j} \sigma_{k}^{z}} \sigma_{j}^{ \pm} q^{\sum_{l>j} \sigma_{l}^{z}}, S^{z}=\sum_{j}^{s} \sigma_{j}^{z}$. Note that at $s=1$, we get an integrable version of an earlier model qbs.

Similarly the same general model (10) with choice $c_{0}=i, c=1$ and realization $s_{q}^{+}=b_{q}^{\dagger}, s_{q}^{-}=$ $b_{q}, s^{3}=N$ yield a new integrable q-deformation of the JC model, while under reduction $c_{0}=i, c=0$ and the same realization through canonical operators as for the TI model, it generates an integrable q-deformation of the TI model. By taking higher $N_{a}$ values multi-atom integrable variants of all the above q-deformed matter-radiation models can be constructed.

We emphasize that all MR models presented here, similar to their unified construction, allow their exact BA solutions also in a unified and almost model-independent way. In BA formalism the diagonal entries $\tau(\lambda)=T_{11}(\lambda)+T_{22}(\lambda)$ produce all conserved operators, while the off-diagonal elements $T_{21}(\lambda) \equiv B(\lambda)$ and $T_{12}(\lambda) \equiv C(\lambda)$ act like creation and annihilation operators of pseudoparticles with the M-particle state defined as $\left|M>_{B}=B\left(\lambda_{1}\right) \cdots B\left(\lambda_{M}\right)\right| 0>$ and the pseudovacuum $\mid 0>$ through $C(\lambda) \mid 0>=0$. The basic idea of algebraic BA aba is to find the eigenvalue solution: $\tau(\lambda)\left|M>_{B}=\Lambda\left(\lambda,\left\{\lambda_{a}\right\}\right)\right| M>_{B}$, for which diagonal elements $T_{i i}(\lambda), i=1,2$ are pushed through the string of $B\left(\lambda_{a}\right)$ 's toward $\mid 0>$, using the commutation relations obtainable from the YB equation. Considering further the actions $T_{11}(\lambda)|0>=\alpha(\lambda)| 0>, T_{22}(\lambda)|0>=\beta(\lambda)| 0>$, one arrives finally at the eigenvalue expression

$$
\Lambda\left(\lambda,\left\{\lambda_{a}\right\}\right)=\alpha(\lambda) \prod_{a=1}^{M} f\left(\lambda-\lambda_{a}\right)+\beta(\lambda) \prod_{a=1}^{M} f\left(\lambda_{a}-\lambda\right),
$$

where $f(\lambda)$ is defined through the elements of the $R$-matrix as $\frac{\lambda+\alpha}{\lambda}$, for the rational and as $\frac{\sin (\lambda+\alpha)}{\sin \lambda}$ for the trigonometric case. Expanding $\Lambda\left(\lambda,\left\{\lambda_{a}\right\}\right)$ in powers of $\lambda$ we obtain the eigenvalues for all conserved operators including the Hamiltonian, where the rapidity parameters $\left\{\lambda_{a}\right\}$ involved can be determined from the Bethe equations

$$
\frac{\alpha\left(\lambda_{a}\right)}{\beta\left(\lambda_{a}\right)}=\prod_{b \neq a} \frac{f\left(\lambda_{b}-\lambda_{a}\right)}{f\left(\lambda_{a}-\lambda_{b}\right)}, \quad a=1,2, \ldots, M,
$$

which follow in turn from the requirement of $\mid M>_{B}$ to be an eigenvector. Returning to our models we find that, the major parts in key algebraic BA relations (11) and (12), described by $R$-matrix elements $f(\lambda)$, depend actually on the class to which the models belong, rather than on an individual model. Therefore, for all standard MR systems including BS, JC and TI models, $f(\lambda)$ is given by its same rational form, while for all q-deformed models like qBS, qJC, qTI etc. by its trigonometric expression. The only model-dependent parts in these equations, expressed through $\alpha(\lambda)$ and $\beta(\lambda)$ are determined from our general Lax operator construction, which for the rational class using (11) and (4) is obtained as

$$
\begin{aligned}
& \alpha(\lambda)=\left(c_{1}^{0}(\lambda+r)+c_{1}^{1}\right) \prod_{j}^{N_{a}}\left(\lambda-s+c_{j}\right), \\
& \beta(\lambda)=\left(c_{1}^{0}(\lambda-r)-c_{2}^{1}\right) \prod_{j}^{N_{a}}\left(\lambda+s+c_{j}\right),
\end{aligned}
$$


where $r=<0\left|s^{3}\right| 0>$ depends on particular realization of (2) and $s=-<0\left|S^{z}\right| 0>$ denotes the atomic spin. (13) yields easily the needed forms for BS, JC and TI models, at the corresponding choices of the parameters like (7), as we have noted above. Similarly, for q-deformed models quantum extension of (13) together with the trigonometric form for $f(\lambda)$ have to be considered. For the solution of TI and qIT models however one has to adopt a bit different approach close to that of the Toda chain faba, since pseudovacuum is difficult to determine for such models.

For deriving physical consequences from our constructions, we consider integrable two-level multiatom BS model with inter-atomic couplings, by taking spin- $\frac{1}{2}$ operator $\vec{S}_{j}=\frac{1}{2} \vec{\sigma}_{j}$ in (8). Using the full strength of the BA method exact solutions for this multi-atom model with arbitrary $N_{a}$ and different $\omega_{a}$ 's can be derived from the same BA relations (11) - (13) for all excitations, by just tuning the parameters involved to their required reduction (17). We demonstrate some novel features in Rabi oscillation and VRS by using the BA solutions in the $N_{a}=2$ atom case of (8). The first excited energy spectrum $E_{1}=\omega_{f}+2 \lambda_{1}$ linked with the cubic Bethe equation (12) gives three distinct real roots along the resonance line $\omega_{f}=\omega_{a}\left(\equiv \omega_{a 1}=\omega_{a 2}\right)$, resulting to a triplet structure in the VRS with splittings in excitation spectrum: $E_{1}=2.02,3.02,4.02$ for $\omega_{f}=3.02, \alpha=1$. Consequently, the Rabi-oscillation becomes involved (see Fig.1a) with three transition frequencies. For small detuning $\delta=\omega_{f}-\omega_{a}$ the roots remain real, while at $\delta=\mp 0.30$ two of them coincides, collapsing the Rabi-splitting to the usual doublet. The excitation spectrum correspondingly reduces to $E_{1}=2.05,3.81$ (degenerate) at subdetuning and to $E_{1}=2.22$ (degenerate), 3.99 at super-detuning points, reducing the Rabi-oscillations to single frequency mode (Fig.1b). Beyond these detuning points two of the roots become complex conjugates, leading to an irregular Rabi-oscillation (Fig.1c).

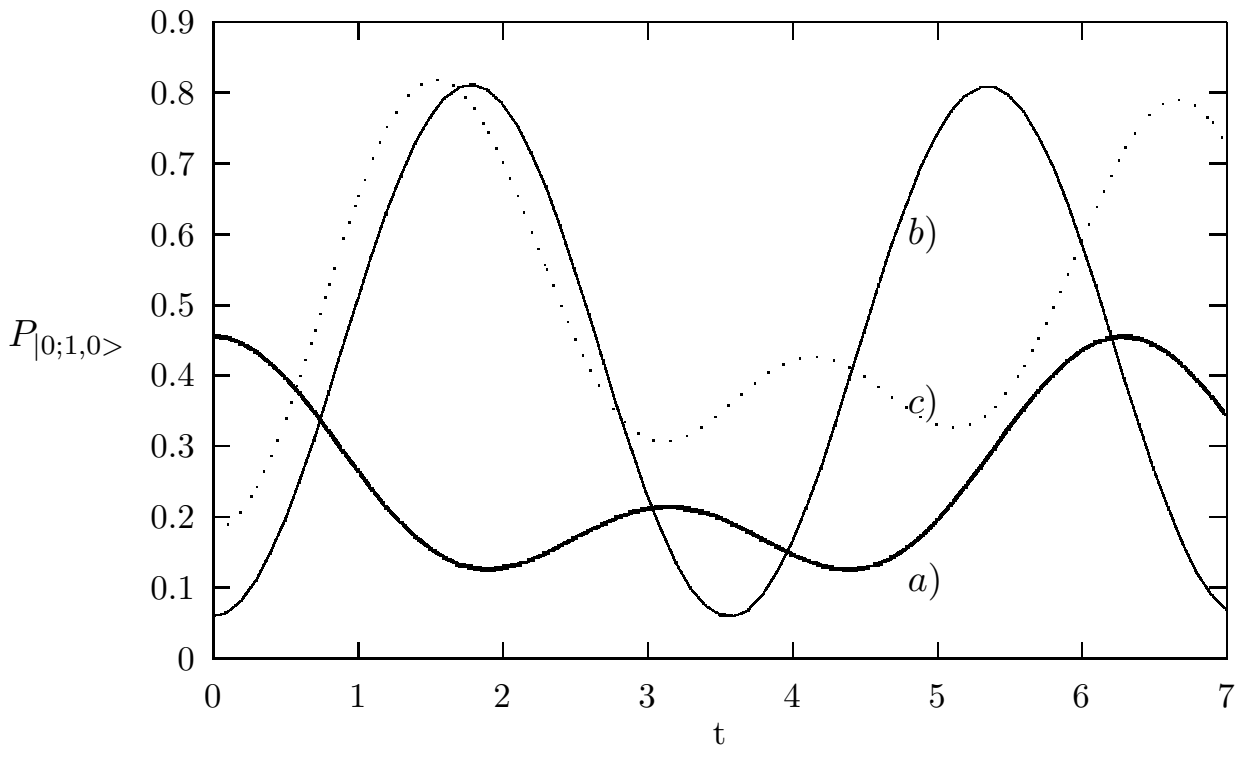

FIG. 1. Time dependence of transition probability, showing Rabi oscillation at different detuning points: at a) resonance, b) degenerate detuning c) further detuning with complex conjugate roots.

Higher excitations for this model as well as its $N_{a}$-atom extension (8) can be solved exactly following the standard BA formalism presented above. We explore now some subtle points and apparent controversies regarding the BA solution of BS models, which also have relevance for other integrable models. A common belief, though proved only for specific models ndBA, is that the degeneracy condition (i.e $\lambda_{a}=\lambda_{b}$ ) for the Bethe states: $\frac{\alpha\left(\lambda_{1}\right)}{\beta\left(\lambda_{1}\right)}= \pm 1$ can not be solved apparently for any integrable model. We however find that for our multi-atom BS model at the resonance point $\delta=\omega_{f}-\omega_{a}=0$, the 
degeneracy condition, which is equivalent to $\alpha\left(\lambda_{1}\right)=\beta\left(\lambda_{1}\right)=0$ is indeed fulfilled, yielding a nontrivial solution $\lambda_{1}=\frac{1}{2}\left(-\omega_{f} \pm 1\right)$, which recovers as well the known spectrum for the standard BS model: $E_{M}=2 M\left(\omega_{f}-\lambda_{1}\right)=M E_{1}$, at resonance bs.

Another apparent controversy regarding multi-atom BS models, which is also generic for many other models but not emphasized properly in the literature, arises due to the fact that, the dimension of the underlying Hilbert space for these models with $N_{a}$ number of two-level atoms has a upper bound $2^{N_{a}}$, for fixed pseudoparticle number. For the standard BS model it is just 2 . Therefore the complexity of the problem can not increase further for higher excitations with $M>N_{a}$. For example, in the BS model by diagonalizing the Hamiltonian directly one can easily get the exact energy spectrum for arbitrary excitation bs . However, when we try to solve the same problem through Bethe ansatz the solution must become increasingly complicated for higher excitations $\mid M>_{B}$, since one has to find all $M$ Bethe roots $\left\{\lambda_{a}\right\}, a=1,2, \ldots, M$ as solutions to general Bethe equations (12), which is impossible analytically! We resolve this problem in an intriguing way by observing that Bethe state $\mid M>_{B}$ and the energy eigenvalues depend in fact not on $M$ number of roots $\lambda_{a}$ individually, but only on some symmetric combinations of them and moreover, the number of these relevant variables does not exceed the dimension of the Hilbert space. For the BS model for example, we find them to be only two: $X_{M}, Y_{M}$ and for deriving them explicitly we introduce an equivalent set of symmetric Bethe roots through symmetric combinations of the original $M$ roots future: $s_{1}=\sum_{a} \lambda_{a}, s_{2}=\sum_{a b}=$ $\lambda_{a} \lambda_{b}, \ldots, s_{M}=\prod_{a} \lambda_{a}$. Combining suitably BAE (12) and (11) for the BS model, expressed through symmetric roots we arrive at the equations

$$
E_{M} X_{M}=\Delta_{+} X_{M}+M Y_{M}, \quad E_{M} Y_{M}=\Delta_{-} Y_{M}+M X_{M},
$$

where $\Delta_{ \pm}=M \omega_{f} \pm \frac{1}{2} \delta$. On the other hand, expressing the Bethe states through two basic states of the model we find $\left|M>_{B}=X_{M}\right| M,->+Y_{M} \mid M-1,+>$, i.e. dependant again on the above two relevant variables only. It is easy to see that the action of the BS Hamiltonian on this eigenstate also reproduces the same relation (14), we have derived from the BAE. Fixing $\omega_{f}=1$ for simplicity, we find these two variables explicitly through symmetric Bethe roots in the form $X_{M}=M s_{M}$ and $Y_{M}=-M s_{M}+\frac{1}{2} \delta\left(s_{M-1}+\ldots+s_{1}+1\right)$. From the first of the relations (14) we get the energy spectrum as $E_{M}=\Delta_{+}+M \kappa_{M}$, where $\kappa_{M}=\frac{Y_{M}}{X_{M}}$ and using both these relations derive the simple equation $M \kappa_{M}^{2}+\delta \kappa_{M}-M=0$. This quadratic equation is solved easily to yield $E_{M}=M \omega_{f} \pm\left(\delta^{2}+M^{2}\right)^{\frac{1}{2}}$, recovering the known spectrum of the BS model in the general $\delta \neq 0$ case. Thus through BA we get the explicit result for all higher excitations, also analytically, resolving the raised controversy. Similar arguments must hold in the corresponding problem for other models.

Thus we have proposed through general Yang-Baxter algebra a series of new integrable multi-atom matter-radiation models including q-deformed models and solved them exactly through Bethe ansatz in a unified way. Integrable trapped ion (TI) and q-deformed TI models introduced here are new, while q-deformed Jaynes-Cummings (JC) and Buck-Sukumar (BS) models are multi-atom as well as integrable extensions of earlier models qbs, qjc. The proposed JC and BS models are nontrivial generalizations of well known models jcbethe,bsbethe, with the inclusion of inter-atomic interactions and nondegenerate atomic frequencies. We find that, contrary to the popular belief, the degenerate Bethe states do exist in the multi-atom BS models at the resonance point. Multi-radiation modes can be included easily in such models preserving integrability. Identifying the models in real systems and experimental verification of the related results presented here, especially in many-atom microlasers 
nØ3, would be an important problem.

\section{References}

[1] G. Rempe, H. Walther and N. Klein , Phys. Rev. Lett. 58 , 353 (1987); G. Rempe, F. Schmidtkaler and H. Walther, Phys. Rev. Lett. 64, 2783 (1990)

[2] M. Raizen et al , Phys. Rev. Lett. 63, 240 (1989)

[3] C. A. Blockley, D. F. Walls and H. Risken, Europhys. Lett. 17, 509 (1992) ;

[4] W. Vogel and R. de Mitos Filho, Phys. Rev. A 52, 4214 (1995)

[5] E. T. Jaynes and F. W. Cummings, Proc. IEEE 51, 89 (1963)

[6] B. Buck and C. V. Sukumar, Phys. Lett. 81 A, 132 (1981)

[7] S. Yu, H. Rauch and Y. Zhang, Phys. Rev. A 52, 2585 (1995)

[8] J. Sanchez-Mondragon, N. B. Narozhny and J. H.Eberly, Phys. Rev. Lett. 51, 550 (1983); R. J. Thompson, G. Rempe and H. J. Kimble, Phys. Rev. Lett. 68, 1132 (1992)

[9] H. S. Zheng, L. M. Kuang, K. L. Gao , Jaynes-Cummings model dynamics in two trapped ions, arXiv:quant-ph/0106020 (2001)

[10] G. S. Agarwal, Phys. Rev. Lett. 53, 1732 (1984)

[11] M. Tavis and F. W. Cummings, Phys. Rev. 170, 379 (1968); W. R. Mallory, Phys. Rev. 188, $1976(1969)$

[12] N. Bogolubov et al, J. Phys. A 29, 6305 (1996)

[13] A. Rybin et al, J. Phys. A 31, 4705 (1998)

[14] A. Kundu, Phys. Rev. Lett. 82, 3936 (1999)

[15] M. Chaichian, D, Ellinas and P. Kulish , Phys. Rev. Lett. 65, 980 (1990)

[16] V. Buzek, J. Mod. Opt. 39, 949 (1992)

[17] L. Takhtajan, Exactly solvable problems in condensed matter and relativistic fiekd theory,(Spriger Verlag, 1985), 175

[18] V. Pasquier and H. Saleur, Nucl Phys. B 330, 523 (1990)

[19] E. K. Sklyanin, Lect. Notes in Phys. (Springer), 226, 196 (1985)

[20] A useful reformulation of Bethe equations achieved through symmetric roots will be presented elsewhere.

[21] A. Izergin and V. Korepin, Lett. Math. Phys. 6, 283 (1982)

[22] K. An et al, Phys. Rev. Lett. 73, 3375 (1994); K. An, J. Phys. Soc. Jpn. 72, 811 (2003) 